\title{
LASER CLEANING OF THE OUTPUT WINDOW IN A LASER IGNITION SYSTEM FOR GAS TURBINES
}

\author{
Paper \#M803
}

\author{
Jonathan Griffiths ${ }^{1}$ and Jonathan Lawrence ${ }^{1}$ \\ ${ }^{1}$ Laser Engineering Group, School of Engineering, University of Lincoln, Brayford Pool, Lincoln, LN6 7TS, \\ UK
}

\begin{abstract}
Laser ignition (LI) of both liquid fuels and gaseous combustible mixtures in gas turbines offers the potential for reduced emissions and increased reliability. During the combustion process, carbon and other by-products accumulate on the walls of the combustion chamber. For laser based ignition systems, this could potentially reduce the transmissive properties of the output window required for transmission of the laser radiation into the combustion chamber. Presented in this paper is an empirical study into the laser cleaning of an output window for the removal of accumulated carbon prior to laser ignition, with the mechanism of removal discussed.
\end{abstract}

\section{Introduction}

In conventional high energy spark ignition (SI), the location in which the ignitor is placed within the combustion chamber is highly detrimental to its durability, reducing its service life. This is primarily due to exposure to the high temperatures and pressures, as well as deposition and accumulation of the products of combustion. The latter not only damages the ignitor but also severely affects the degree to which the fuel spray can be atomized by the fuel injection lance, necessitating the use of higher energy density plasmas for ignition thus exacerbating the problem. The combined adverse effects attributable to erosion, or the significant deposition and accumulation of the products of combustion, may affect successful ignition or compromise component integrity to such an extent that the high energy ignitor may need to be replaced prematurely.

Laser based ignition (LI) offers the potential to physically remove the ignitor assembly from the combustion chamber, thereby addressing the majority of the aforementioned durability issues. However, an output window is required for transmission of the beam into the combustion chamber and this would still be subject to accumulation of debris from the combustion process, adversely affecting the transmission properties of the window [1]. This has also been identified as an issue in optical monitoring of combustion events [2]. A self-cleaning mechanism [3] has been reported in literature regarding LI, the principles of which are broadly the same as those of selective vaporization and spallation, both widely applied laser cleaning mechanisms [4].

Previously, the ability to vary the ignition location freely within the combustion chamber has been cited as an inherent advantage of laser based ignition systems when compared with conventional SI [4-6]. However, the aforementioned self-cleaning process is heavily dependent on the energy density of the beam incident on the window. Therefore, both the position of the window in the optical path as well as laser operating parameters must be chosen so as to facilitate self-cleaning prior to $\mathrm{LI}$.

In this paper an empirical study into the removal of a carbon layer from the reverse side of a borosilicate glass output window using a Q-switched Nd:YAG laser source is detailed, with the effect of focal position and laser power on the effectiveness of removal investigated

\section{Experimental}

Laser cleaning of a $75 \times 25 \times 1 \mathrm{~mm}^{3}$ borosilicate glass output window was investigated using a Q-switched $\mathrm{Nd}$ :YAG TEM $\mathrm{T}_{00}$ laser (Powerlite II, Continuum Ltd), operating at $10 \mathrm{~Hz}$ repetition rate and $532 \mathrm{~nm}$ wavelength. The experimental set-up for the output window cleaning investigation is depicted Figure 1.

The set-up consisted of a Galilean beam expander, reflective beam steering mirrors and a polarization based variable attenuator. The beam was focussed onto the output window using a $200 \mathrm{~mm}$ focal length $\mathrm{N}$ BK7 plano-convex lens. A power meter was placed approximately $40 \mathrm{~mm}$ after focal position in the optical path.

Borosilicate glass was chosen as a cost effective and suitable approximation of N-BK7 glass, a likely 
candidate for use in LI systems utilizing visible wavelengths. The transmission of $532 \mathrm{~nm}$ radiation in borosilicate and N-BK7 glass is $98.75 \%$ and $99.5 \%$ respectively. Whilst the thickness of the borosilicate glass used $(1 \mathrm{~mm})$ was considerably less than that of output window in the ignitor assembly $(5 \mathrm{~mm})$ this was likely to have little effect on the results of this investigation due to the highly transmissive nature of both.

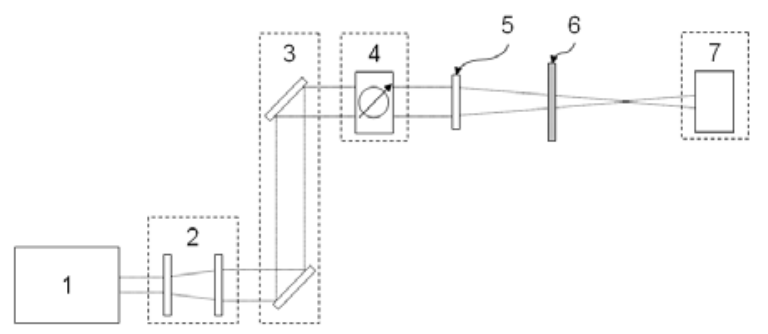

Figure 1: Output window cleaning experimental set-up with (1) laser source, (2) Galilean beam expander, (3)

beam steering mirrors, (4) variable attenuator, (5)

focussing lens, (6) graphite coated output window and (7) power meter.

The output window was coated with graphite spray to simulate the accumulation of debris (predominantly carbon) observed on the walls of the combustion chamber during gas turbine operation. The average layer thickness was determined using white light interferometry (WLI, NewView 700s, Zygo Corp.) and found to be approximately $0.15 \mathrm{~mm}$. The uncoated side of the window was exposed to laser radiation.

\section{Results}

The ability of pulsed laser radiation to remove a 0.15 $\mathrm{mm}$ thick carbon layer from the reverse side of $75 \times 25$ x $1 \mathrm{~mm}^{3}$ borosilicate glass output window was investigated using a the Powerlite system; operating at $10 \mathrm{~Hz}$ repetition rate and $532 \mathrm{~nm}$ wavelength. The laser power and position of the output window in the optical path were varied, with the input and transmitted power measured.

\section{Operating window}

Initially, the laser power required for spark formation for this optical set-up was determined. Operating at 10 $\mathrm{Hz}$ repetition rate, the laser power was gradually increased until consistent sparking was observed, with the transmitted power through the focal point measured using a power meter. It should be noted that, for this part of the investigation, the output window was removed from the experimental set-up depicted in Figure 1. Sparking was found to occur at incident powers of $70 \mathrm{~mW}$ or greater, with consistent sparking occurring at approximately $100 \mathrm{~mW}$.

This region of infrequent spark formation observed from 70 to $100 \mathrm{~mW}$ was also observed by Chen et al [8]. To investigate this further, the laser was set to single pulse mode, with 100 pulses triggered at each power over this region. The number of sparks generated was monitored and used to determine the spark probability, as shown in Figure 2 along with transmitted intensity through the focal position.

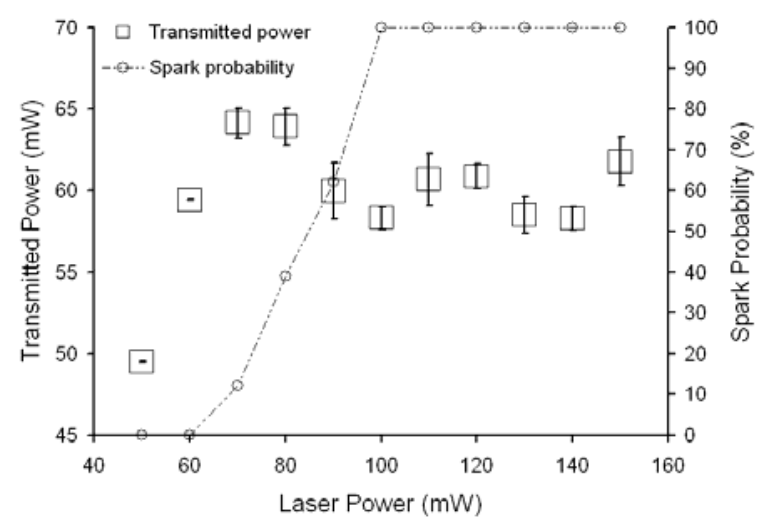

Figure 2: Transmitted laser power and spark probability with increasing laser power $(532 \mathrm{~nm}, 10$

$\mathrm{Hz})$.

\section{Output window cleaning}

The transmitted power through a graphite coated output window at 50, 150, 250, 350, 450 and $550 \mathrm{~mW}$ input powers was measured using the experimental setup depicted in Figure 1. This represented the full range of powers available at $532 \mathrm{~nm}$ wavelength using the Powerlite system.

The output window was placed $10 \mathrm{~mm}$ from the focal point of a $200 \mathrm{~mm}$ lens, equivalent to the position it would occupy should it be incorporated as part of the pilot burner assembly and the spark be located in the same position as that of the high energy ignitor spark in conventional SI. The results are shown in Figure 3.

It was found that, with the window at this position in the optical path, even powers below that required for spark formation $(<70 \mathrm{~mW})$ were sufficient for the complete removal of the carbon layer. Figure 3 reveals near full transmission of the input laser power at 50 $\mathrm{mW}$, with losses likely to be due predominantly to reflection at the input face of the window. It should be noted that the output window in an LI system could be anti-reflective coated in order to reduce such losses. 


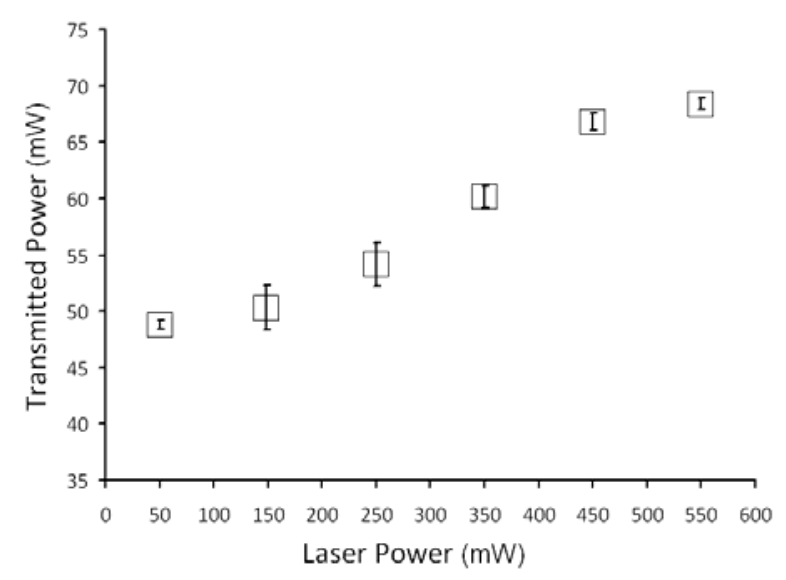

Figure 3: Transmitted power through graphite coated output window with varying laser power at $10 \mathrm{~mm}$ from the focal position $(532 \mathrm{~nm}, 10 \mathrm{~Hz})$.

At powers above the threshold for spark formation the transmitted power was no longer directly proportional to the input power. This is due to the partial absorption of the beam in the plasma generated in the focal position. After dielectric breakdown of air has been initiated, the electrons released as a result of this ionization readily absorb more of the incident photons via inverse bremsstrahlung, as shown in Figure 4.

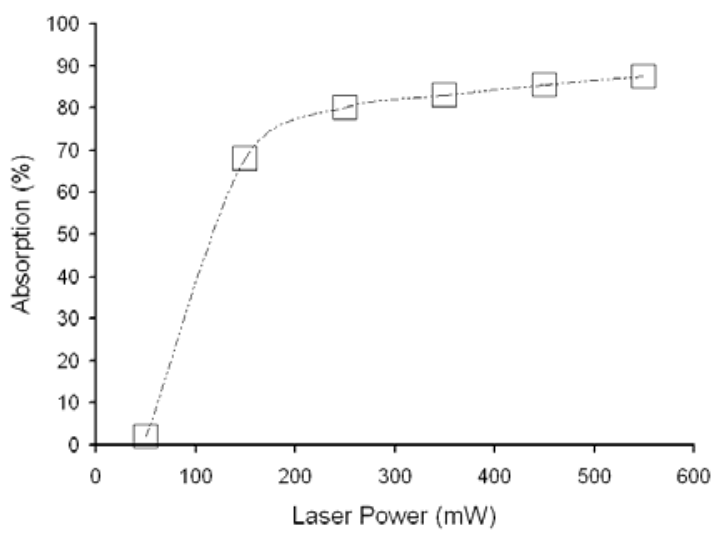

Figure 4: Absorption of incident laser radiation in the in the plasma generated with increasing laser power $(532 \mathrm{~nm}, 10 \mathrm{~Hz})$.

Fluctuation in transmitted power was found to decrease with increasing incident power. This is likely due to saturation of the laser generated plasma. Increasing incident power corresponds to an increase in the number of photons incident on the plasma. A region in which the energy and density of the plasma is increased exists between the threshold for spark formation and the saturation point. Beyond this point the plasma expands in size without significant change in density [9] and only the trailing edge of the laser pulse is transmitted through the focal position. Therefore, the transmitted power shows a linear relationship with incident power after saturation of the plasma has been achieved.

The position of the output window in the optical path is crucial to the ability of the laser to remove the carbon layer effectively. With increasing proximity to the focal position, the laser intensity is sufficient for full removal of the layer, whilst further away from the focal position only partial removal of the layer is possible, as depicted in Figure 5.

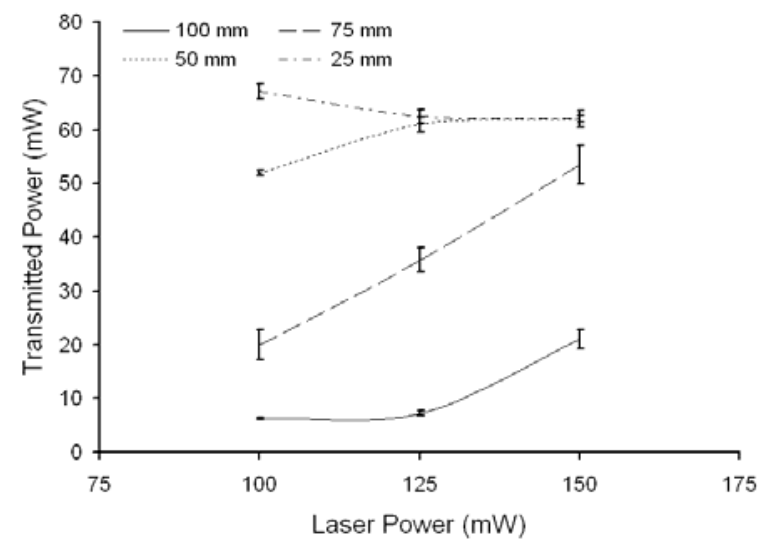

Figure 5: Transmitted power through graphite coated output window with varying laser power and distance from focal position $(532 \mathrm{~nm}, 10 \mathrm{~Hz})$.

At least partial removal of the carbon layer was achieved at all the powers and distances used in Figure 5. Spark formation occurred at all powers when the window was placed within $50 \mathrm{~mm}$ of the focal position. The distance from the focal position at which full removal of the carbon layer is possible is dictated by the focal length of the lens, beam diameter incident on the lens and laser power.

\section{Mechanism of cleaning}

The mechanism by which the carbon layer is removed is likely a combination of two well established mechanism of laser cleaning in conservation of artworks; spallation and selective vaporization [10].

Spallation utilizes high laser intensities, such as those generated by Q-switched solid state lasers, at which even reflective materials absorb strongly enough for vaporization to occur. The plasma generated by this initial vaporization has the effect of shielding the rest of the substrate from the laser radiation by absorbing it through the mechanism of inverse bremstrahlung. Absorption of the laser radiation causes the plasma to expand, imparting compressive stresses into the 
substrate. After the laser pulse has ended the plasma rapidly expands away from the substrate surface, resulting in the relaxation of the compressive stresses and the removal of a layer up to $100 \mu \mathrm{m}$ thick.
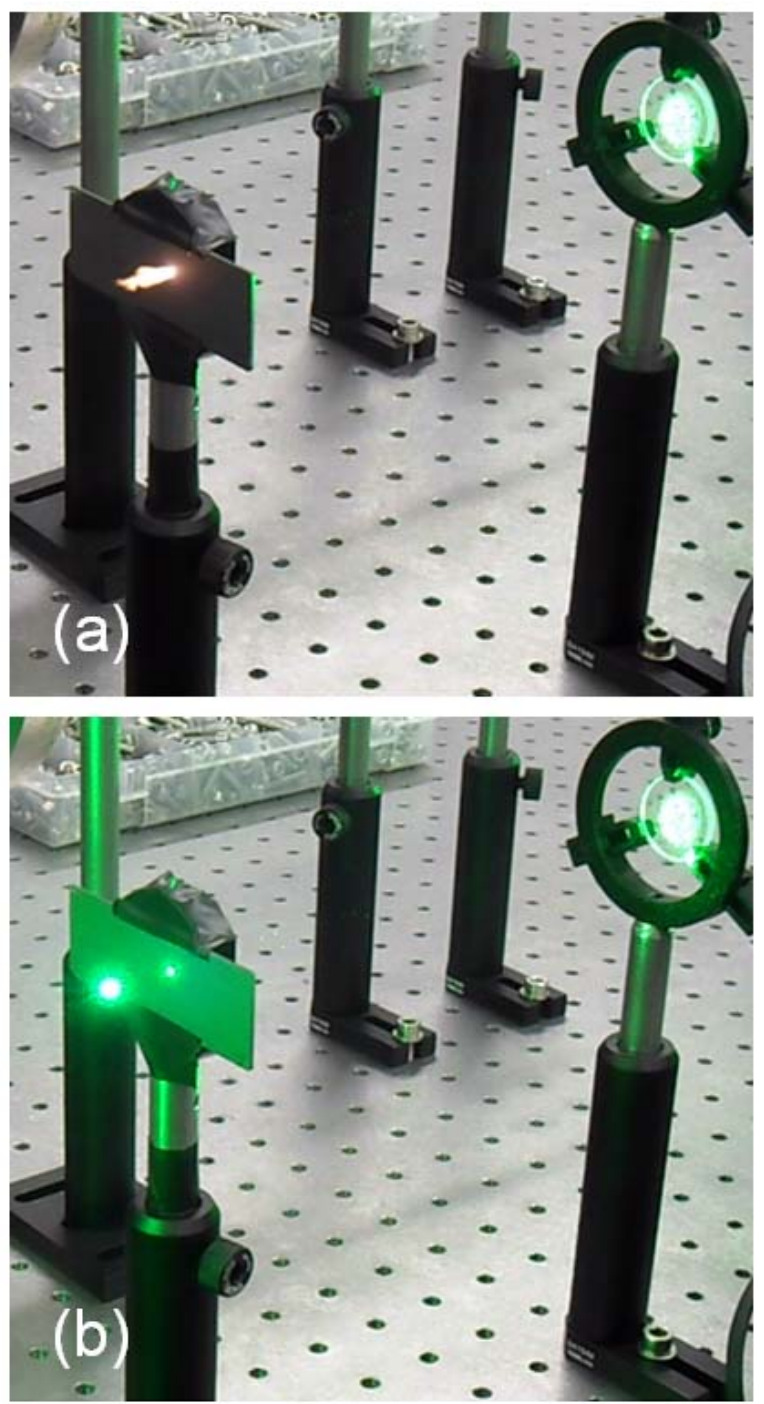

Figure 6: Output window cleaning showing (a) initial pulse removing graphite coating from window and (b) subsequent pulse resulting in spark formation $(50 \mathrm{~mW}$, $532 \mathrm{~nm}, 10 \mathrm{~Hz}, 10 \mathrm{~mm}$ from focal position).

In the selective vaporization process, a difference in the reflectivity of a substrate and debris layer results in strong absorption in one but no appreciable temperature rise in the other. This allows for the selective vaporisation of debris layers, especially if they are dark coloured and the substrate is particularly reflective. However, as previously stated, the use of high intensities would likely result in damage of the substrate, regardless of its reflectivity. Whilst the high laser intensities used in this investigation would likely rule out the selective vaporization mechanism for removal of the carbon layer from opaque substrates, the highly transmissive nature of the borosilicate glass used allows this mechanism to operate without damaging the substrate. As depicted in Figure 6, the first pulse removes the carbon layer from the reverse side of the glass by a process of selective vaporization, with little or no transmission of power and no spark generated. Subsequent pulses are then fully transmitted and result in spark formation at the focal position.

As previously mentioned, minimal or no damage to the substrate is indicative of selective vaporization being the active mechanism of laser cleaning. Figure 7 shows no appreciable damage to the surface of a laser cleaned output window when compared with an un-processed sample.

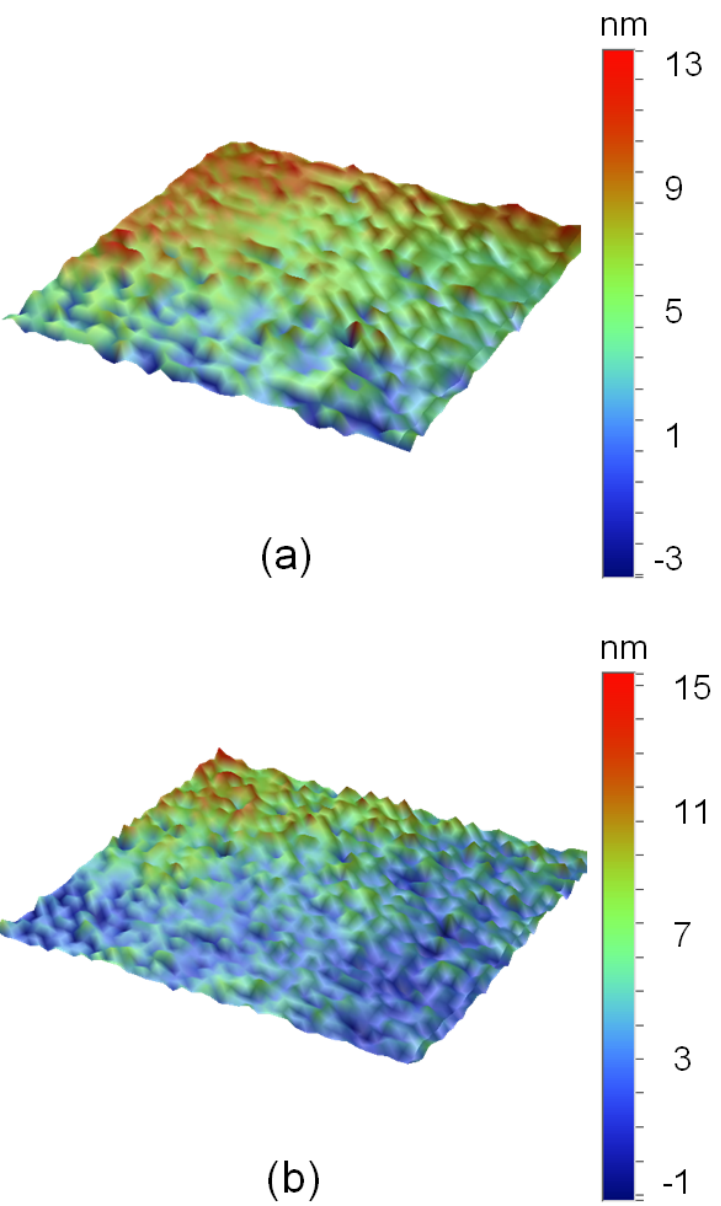

Figure 7: WLI image of an approximately $80 \times 80 \mu \mathrm{m}$ central portion of borosilicate glass surface (a) before laser cleaning and (b) after $(100 \mathrm{~mW}, 532 \mathrm{~nm}, 10 \mathrm{~Hz}$, $10 \mathrm{~mm}$ from focal position). 


\section{Conclusions}

Laser cleaning of carbon deposits on the output window of the ignitor assembly has been successfully demonstrated. A full empirical study was conducted in which the laser power and position of the output window were varied in order to determine a suitable processing window for laser ignition (LI).

It was found that the position of the output window in the optical path was paramount to the suitability of operating parameters for a given optical system. The key operating parameters were identified as focal length of the lens, beam diameter incident on the lens and laser power. This can be attributed to the variation in the energy density of the beam throughout its focal length. Therefore, the configuration of the optical system is an important consideration when selecting ignition location within the combustion chamber.

\section{References}

[1] Graf J, Weinrotter M, Kopecek H, Wintner E. Laser Ignition, Optics and Contamination of Optics in an I.C. Engine. ASME Conference Proceedings 2004;2004(37467):11-17.

[2] Hirvonen J, Pekka K, Takala M, inventors. Imatran Voima Oy, assignee. Assembly for Combustion Chamber Monitoring Camera. Finland patent 5592217. 1993 Jan. 7, 1997.

[3] Mullett JD, Dearden G, Dodd R, Shenton AT, Triantos G, Watkins KG. A comparative study of optical fibre types for application in a laser-induced ignition system. Journal of Optics A: Pure and Applied Optics 2009; 11(5):054007.

[4] Weinrotter M, Kopecek H, Tesch M, Wintner E, Lackner M, Winter F. Laser ignition of ultra-lean methane/hydrogen/air mixtures at high temperature and pressure. Exp Therm Fluid Sci 2005 6; 29(5):569577.

[5] Morsy MH, Ko YS, Chung SH. Laser-induced ignition using a conical cavity in CH4-air mixtures. Combust Flame 1999 12; 119(4):473-482.

[6] Morsy MH, Chung SH. Laser-induced multi-point ignition with a single-shot laser using two conical cavities for hydrogen/air mixture. Exp Therm Fluid Sci 2003 4; 27(4):491-497.

[7] Watkins KG, Curran C, Lee J. Two new mechanisms for laser cleaning using Nd:YAG sources. Journal of Cultural Heritage 2003 1; 4, Supplement 1(0):59-64.
[8] Chen Y, Lewis JWL, Parigger C. Spatial and temporal profiles of pulsed laser-induced air plasma emissions. Journal of Quantitative Spectroscopy and Radiative Transfer 2000 10/16; 67(2):91-103.

[9] Yalcin S, Crosley D, Smith G, Faris G. Spectroscopically Determined Temperatures and Electron Densities in Laser Produced Sparks. Int OSA Annual Meeting Technical Digest 1995:147.

[10] Steen WM, Mazumder J. Laser Materials Processing. 6th ed.: Springer; 2010.

\section{Acknowledgements}

The author would like to thank Siemens Industrial Turbomachinery Ltd (SITL) for funding this investigation as part of a wider investigation into laser ignition for gas turbines.

\section{Meet the Author}

Jonathan Griffiths is a research fellow in the Laser Engineering Group within the School of Engineering at the University of Lincoln. His main research area is laser ignition for gas turbines. He has presented his work at a number of international conferences and has published three journal papers to date. 